\title{
Efficacy of Androgen Deprivation Therapy in Patients with Metastatic Castration-Resistant Prostate Cancer Receiving Docetaxel-Based Chemotherapy
}

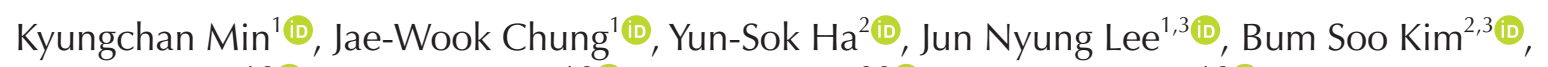

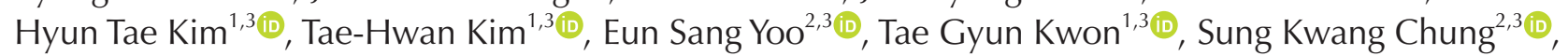
Masatoshi Tanaka ${ }^{4}$ (DiD, Shin Egawa ${ }^{4}$ (iD), Takahiro Kimura ${ }^{4, *}$ (iD), Seock Hwan Choi ${ }^{2,3, *(\mathbb{D})}$

${ }^{1}$ Department of Urology, Kyungpook National University Chilgok Hospital, ${ }^{2}$ Department of Urology, Kyungpook National University Hospital, ${ }^{3}$ Department of Urology, School of Medicine, Kyungpook National University, Daegu, Korea, ${ }^{4}$ Department of Urology, Jikei University School of Medicine, Tokyo, Japan

Purpose: The purpose of this study was to determine the comparative effectiveness of androgen deprivation therapy (ADT) combined with docetaxel (DTX)-based chemotherapy in Korean and Japanese castration-resistant prostate cancer (CRPC) patient cohorts.

Materials and Methods: Metastatic CRPC patients who underwent more than three DTX-based chemotherapy cycles in Korea and Japan between 2002 and 2017 were retrospectively analyzed and divided into the DTX-only (DTX, n=30) and combination (DTX+ADT, n=46) groups. Progression-free survival (PFS) was calculated as the time from the start of chemotherapy to the occurrence of either disease progression (prostate-specific antigen [PSA] progression or radiographic progression) or death. The primary end point was PFS and the secondary end point was overall survival (OS).

Results: In the DTX and DTX+ADT groups, the median PFS was 6.0 and 11.0 months (log-rank $p=0.053$ ). The multivariate Cox regression analysis revealed that the significant predicting factors of PFS were ADT administration (hazard ratio [HR], $0.478 ; 95 \%$ confidence interval $[\mathrm{Cl}], 0.284-0.804 ; \mathrm{p}=0.005)$ and number of DTX-based chemotherapy cycles $(\mathrm{HR}$, 0.934 ; 95\% Cl, 0.899-0.970; $\mathrm{p}<0.001)$. In the DTX and DTX+ADT groups, the median OS was 16.0 and 19.5 months (log-rank $\mathrm{p}=0.825$ ). Through multiple Cox regression analysis, we found that the significant predicting factors of OS were the PSA nadir level $(\mathrm{HR}, 1.001 ; 95 \% \mathrm{Cl}, 1.000-1.002 ; \mathrm{p}<0.001)$ and number of DTX-based chemotherapy cycles $(\mathrm{HR}, 0.932 ; 95 \% \mathrm{Cl}$, $0.876-0.991 ; p=0.024)$.

Conclusions: Concurrent DTX-based chemotherapy and ADT may be beneficial compared with DTX-based chemotherapy alone in chemotherapy-naïve metastatic CRPC patients in terms of the PFS, but not the OS.

Keywords: Antineoplastic hormonal drugs; Docetaxel; Progression-free survival; Prostate cancer

This is an Open Access article distributed under the terms of the Creative Commons Attribution Non-Commercial License (http://creativecommons.org/licenses/by-nc/4.0) which permits unrestricted non-commercial use, distribution, and reproduction in any medium, provided the original work is properly cited.

Received: Mar 2, 2019 Revised: May 3, 2019 Accepted: May 13, 2019 Published online Jun 4, 2019

Correspondence to: Seock Hwan Choi (iD https://orcid.org/0000-0003-3796-2601

Department of Urology, School of Medicine, Kyungpook National University, 680 Gukchaebosang-ro, Jung-gu, Daegu 41944, Korea.

Tel: +82-53-200-5855, Fax: +82-53-421-9618, E-mail: skhwan@gmail.com

*These authors contributed equally to this study. 


\section{INTRODUCTION}

Prostate cancer is the most frequently diagnosed cancer and second most frequent cause of cancer-related death among men in the USA [1]. Similar growing trend has been reported in Asian countries, especially those in the northeast region [2]. In Korea, it is ranked as the fifth newly diagnosed cancer, and seventh cause of cancer-related death in men; showing rapid increase [3]. In Japan, prostate cancer was ranked as the leading cancer type among men in 2015 [4]. In patients with metastatic and locally advanced prostate cancer, androgen deprivation therapy (ADT) is extremely effective initially, but all patients undergoing the treatment develop resistance to ADT [5]. Recently, clinical investigations focusing on non-hormonal approaches revealed that systemic chemotherapy including docetaxel (DTX) improved the life expectancy and quality of life in castration-resistant prostate cancer (CRPC) patients [6,7].

The effectiveness of continuing ADT to prolong life expectancy in patients with CRPC is controversial $[8,9]$. The general consensus is to maintain all patients on repetitive dosage of luteinizing hormone-releasing hormone (LHRH) agonist or antagonist. Two early trials have shown only marginal survival benefit in patients administered LHRH analogs as part of second- and third-line therapies. However, prospective data are lacking, and studies of all subsequent treatments included men with ongoing androgen suppression. All clinical trials of new drugs for men with CRPC have mandated the continued suppression of serum testosterone levels, either with continuous ADT or with surgical castration. However, there is no conclusive evidence for the efficacy of concomitant ADT in patients with CRPC.

Androgen receptors (ARs) maintain active function despite frequent alterations in the patients' CRPC status [10]. We hypothesized that concurrent ADT and DTX-based chemotherapy yields no survival benefit compared with DTX-based chemotherapy alone in metastatic CRPC patients, based on evidence of testicular suppression for approximately 6 to 7 months after ADT. Until recently, concurrent DTX-based chemotherapy and ADT was contraindicated in metastatic CRPC patients in Korea, unlike the indication in several other countries, including Japan. The purpose of this study was to determine the comparative effectiveness of ADT combined with DTX-based chemotherapy in Korean and Japanese patient cohorts with CRPC.

\section{MATERIALS AND METHODS}

The study cohorts comprised a total of 141 patients with CRPC who were chemotherapy-naïve. Patients from two tertiary hospitals in Korea and one hospital in Japan who were treated with more than three cycles of DTX-based chemotherapy between 2002 and 2017 were included and retrospectively analyzed. Finally, a total of 76 patients who met the following criteria were enrolled: 1) pathologic confirm of prostatic adenocarcinoma, 2) presence of metastasis at DTX-based chemotherapy induction, 3) Eastern Cooperative Oncology Group performance status $\leq 2,4$ ) without prior treatment with mitoxantrone or radioisotope, 5) without evidence of metastasis in the central nervous system, and 6) adequate bone marrow and organ function.

During the study period, the Korean patients with metastatic CRPC were unable to receive concurrent DTX-based chemotherapy and ADT. Thus, the Korean cohort included patients who were treated with DTXbased chemotherapy alone (DTX group, $n=30$ ). In contrast, Japanese patients were allowed concurrent DTXbased chemotherapy and ADT. Therefore, the Japanese cohort included patients who were treated with the combined therapy (DTX+ADT group, $\mathrm{n}=46$ ).

\section{Treatment and evaluation}

DTX-based chemotherapy and prednisolone therapy with or without ADT was administered in all patients. The DTX-based chemotherapy regimen consisted of intravenous DTX at day 1 every 3 weeks, with oral prednisolone $5 \mathrm{mg}$ twice daily starting on day 1 and be continued throughout the treatment. The intravenous infusion dose was adjusted according to the patients' general condition and results of laboratory studies. The average dose was $75 \mathrm{mg} / \mathrm{m}^{2}$ per cycle. In almost all the patients of DTX+ADT group, ADT regimen consisted of trimonthly LHRH agonist injection with daily oral antiandrogen medication. In the rest of the patients of DTX+ADT group, ADT regimen was trimonthly LHRH agonist injection only or daily oral antiandrogen medication only.

DTX-based chemotherapy was continued until prostate-specific antigen (PSA) progression, clinical progression, adverse events, patient's or physician's decision, or death. PSA progression was defined as the date at which a $\geq 25 \%$ increase and an absolute $\geq 2 \mathrm{ng} / \mathrm{mL}$ increase from the nadir were observed in accordance 
with the Prostate Cancer Working Group (PCWG)-2 criteria. The PSA nadir was defined as the lowest PSA level achieved during DTX-based chemotherapy. Development of more than two new lesions was considered as progression on bone scan. Soft-tissue progression was evaluated in accordance with the Response Evalua- tion Criteria In Solid Tumors version 1.1 [5]. Laboratory studies, including PSA level, complete blood counts, liver function tests and renal function tests were performed at each cycle. Responses were assessed using computed tomography scan at every 3 months or under suspicion of clinical progression.

Table 1. Baseline patient characteristics

\begin{tabular}{|c|c|c|c|}
\hline Characteristic & DTX $(n=30)$ & DTX+ADT $(n=46)$ & $\mathrm{p}$-value \\
\hline Age (y) & $69.43 \pm 8.14$ & $65.93 \pm 8.84$ & 0.086 \\
\hline Gleason score & & & 0.644 \\
\hline$\leq 7$ & $2(6.7)$ & $5(10.9)$ & \\
\hline 8 & $12(40.0)$ & $10(21.7)$ & \\
\hline 9 & $12(40.0)$ & $21(45.7)$ & \\
\hline 10 & $3(10.0)$ & $7(15.2)$ & \\
\hline Unknown & $1(3.3)$ & $3(6.5)$ & \\
\hline PSA level at diagnosis & $151.62 \pm 269.93$ & $591.79 \pm 1942.74$ & 0.137 \\
\hline Clinical T stage at diagnosis & & & $0.001^{*}$ \\
\hline 1 & 0 & $2(4.3)$ & \\
\hline 2 & $5(16.7)$ & $24(52.2)$ & \\
\hline $3 a$ & $10(33.3)$ & $11(23.9)$ & \\
\hline $3 b$ & $11(36.7)$ & $7(15.2)$ & \\
\hline 4 & $4(13.3)$ & 0 & \\
\hline Unknown & 0 & $2(4.3)$ & \\
\hline Clinical N stage at diagnosis & & & $<0.001^{*}$ \\
\hline 0 & $7(23.3)$ & $38(82.6)$ & \\
\hline 1 & $23(82.6)$ & $8(17.4)$ & \\
\hline Clinical M stage at diagnosis & & & 0.695 \\
\hline 0 & $9(30.0)$ & $19(41.3)$ & \\
\hline $1 a$ & $1(3.3)$ & $2(4.3)$ & \\
\hline $1 b$ & $17(56.7)$ & $19(41.3)$ & \\
\hline $1 c$ & $3(10.0)$ & $6(13.0)$ & \\
\hline Definitive local treatment & & & 0.065 \\
\hline No & $25(83.3)$ & $28(60.9)$ & \\
\hline Radical prostatectomy & $4(13.3)$ & $9(19.6)$ & \\
\hline Radiation treatment & $1(3.3)$ & $9(19.6)$ & \\
\hline PSA level at DTX-based chemotherapy induction & $124.34 \pm 230.76$ & $160.66 \pm 585.96$ & 0.748 \\
\hline Clinical N stage at DTX-based chemotherapy induction & & & 0.056 \\
\hline 0 & $23(76.7)$ & $25(54.3)$ & \\
\hline 1 & $7(23.3)$ & $21(45.7)$ & \\
\hline Clinical M stage at DTX-based chemotherapy induction & & & 0.094 \\
\hline $1 a$ & $3(10.0)$ & $2(4.3)$ & \\
\hline $1 b$ & $26(86.7)$ & $35(76.1)$ & \\
\hline $1 c$ & $1(3.3)$ & $9(19.6)$ & \\
\hline PSA nadir level responding to DTX & $48.23 \pm 142.32$ & $125.46 \pm 488.91$ & 0.403 \\
\hline No. of DTX-based chemotherapy cycles (times) & $13.0 \pm 8.9$ & $9.9 \pm 5.2$ & 0.094 \\
\hline ADT before DTX induction (mo) & $31.9 \pm 18.5$ & $45.5 \pm 39.2$ & 0.100 \\
\hline
\end{tabular}

Values are presented as mean \pm standard deviation or number (\%).

DTX: docetaxel, ADT: androgen deprivation therapy, PSA: prostate-specific antigen.

${ }^{*} p<0.05$. 


\section{Study end points}

The majority of tumor burden in metastatic prostate cancer is found in the bones. Hence, the response to treatment at soft-tissue sites alone may not reflect the main treatment benefits because it is representative of only a small proportion of the overall disease burden [5]. In this study, the primary end point of this study was progression-free survival (PFS), and the secondary end point was overall survival (OS).

\section{Statistical analysis}

Chi-square test or Fisher's exact test were used for categorical variables, and paired T-test or Mann-Whitney U-test were used for continuous variables. KaplanMeier method and log-rank test was used for the calculation and analysis of PFS and OS. Cox proportional hazards models were used to perform univariate and multivariate analyses. Factors associated with progression or mortality with a p-value $<0.25$ on univariate analyses were entered in the multivariate model. PFS was calculated as the time from the start of chemotherapy to the occurrence of either disease progression (biochemical recurrence or radiographic progression) or death. OS was defined as the time from the start of chemotherapy to death from any cause. All tests were two-sided, and p-value $<0.05$ was considered to be statistically significant. Statistical Package for the Social Sciences (SPSS) ver. 18 (PASW Statistics; IBM Corp., Armonk, NY, USA) was used for statistical analysis.

\section{Ethics statement}

The present study protocol was reviewed and approved by the Institutional Review Board of Kyungpook National University Hospital (Reg. No. 2019-01010-002).

\section{RESULTS}

The patients' baseline characteristics are summarized in Table 1. We compared several factors between the groups: age, Gleason score, clinical TNM stage at the time of cancer diagnosis, DTX-based chemotherapy induction, type of definitive local treatment, type of ADT protocol, PSA level at each state, number of DTX-based chemotherapy cycles, and ADT period before DTX induction. There were no significant intergroup differences of all factors including clinical $\mathrm{N}$ and $\mathrm{M}$ stages at the time of DTX chemotherapy induction, except for

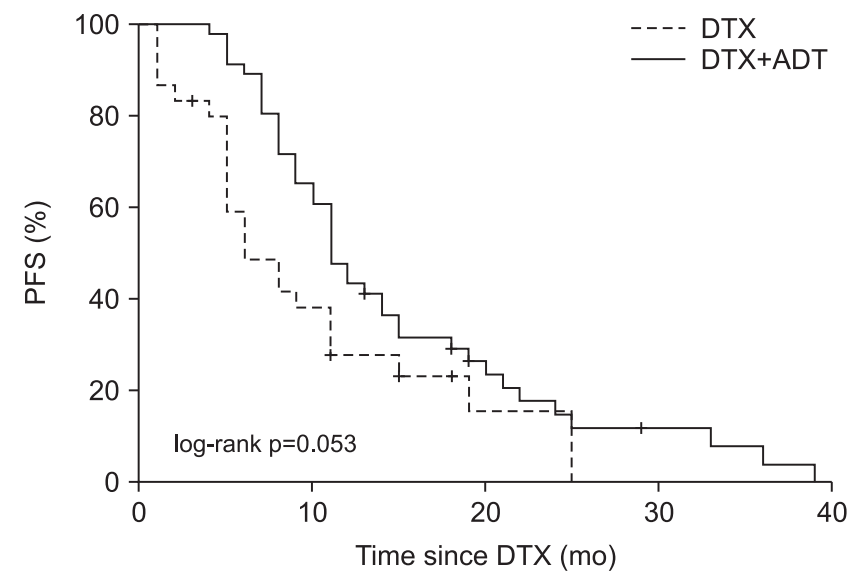

Fig. 1. The dotted line shows the PFS of the DTX group, and the linear line shows the PFS of the DTX+ADT group. PFS: progression-free survival, DTX: docetaxel, ADT: androgen deprivation therapy.

the clinical $\mathrm{T}$ and $\mathrm{N}$ stages at initial cancer diagnosis. There were no differences in the PSA level at each step and number of DTX doses.

Comparison of the PFS between the two groups using Kaplan-Meier analysis is shown in Fig. 1. The median PFS of the DTX group was 6.0 months (95\% confidence interval [CI], 2.86-9.14 months), and that of the DTX+ADT group was 11.0 months (95\% CI, 9.34-12.66 months) (log-rank $\mathrm{p}=0.053$ ). Table 2 summarizes the findings of univariate and multivariate Cox regression analyses of the factors affecting the PFS. Significant factors determined through the univariate analysis including administration of ADT, age, PSA level at DTX-based chemotherapy induction, PSA nadir level and number of DTX-based chemotherapy cycles were included in multivariate analysis. The result obtained through multivariate analysis indicated that the administration of ADT (hazard ratio [HR], 0.478; 95\% CI, $0.284-0.804 ; p=0.005$ ) and number of DTX-based chemotherapy cycles (HR, 0.934; 95\% CI, 0.899-0.970; p<0.001) were significant.

Comparison of the OS between two groups using Kaplan-Meier analysis is shown in Fig. 2. The median OS of the DTX group was 16.0 months (95\% CI, 13.04-18.97 months), and that of the DTX+ADT group was 19.5 months (95\% CI, 15.98-23.02 months) (log-rank p=0.825). Table 3 summarizes the findings of univariate and multivariate Cox regression analyses of the factors affecting the OS. There was no significant difference in the OS between the two groups according to administration of ADT. Significant factors determined through the univariate analysis including Gleason score, PSA 
Table 2. Univariate and multivariate Cox regression analyses of the factors predicting the PFS

\begin{tabular}{|c|c|c|c|c|}
\hline \multirow{2}{*}{ Characteristic } & \multicolumn{2}{|c|}{ Univariate analysis } & \multicolumn{2}{|c|}{ Multivariate analysis } \\
\hline & HR $(95 \% \mathrm{Cl})$ & p-value & $\mathrm{HR}(95 \% \mathrm{CI})$ & p-value \\
\hline ADT & $0.618(0.369-1.035)$ & 0.067 & $0.478(0.284-0.804)$ & $0.005^{*}$ \\
\hline Age & $0.982(0.956-1.008)$ & 0.174 & $0.989(0.961-1.018)$ & 0.446 \\
\hline Gleason score & & 0.699 & & \\
\hline$\leq 7$ & 1.000 (Reference) & & & \\
\hline 8 & $1.185(0.436-3.219)$ & 0.740 & & \\
\hline 9 & $1.649(0.638-4.262)$ & 0.302 & & \\
\hline 10 & $1.168(0.381-3.582)$ & 0.786 & & \\
\hline Unknown & $1.062(0.205-5.517)$ & 0.943 & & \\
\hline PSA level at diagnosis & $1.000(1.000-1.000)$ & 0.424 & & \\
\hline Clinical T stage at diagnosis & & 0.981 & & \\
\hline 1 & 1.000 (Reference) & & & \\
\hline 2 & $1.214(0.285-5.171)$ & 0.793 & & \\
\hline $3 a$ & $1.499(0.346-6.488)$ & 0.682 & & \\
\hline $3 b$ & $1.425(0.321-6.326)$ & 0.642 & & \\
\hline 4 & $1.465(0.242-8.863)$ & 0.678 & & \\
\hline Unknown & $1.510(0.210-10.858)$ & 0.682 & & \\
\hline Clinical $\mathrm{N}$ stage at diagnosis & & 0.703 & & \\
\hline 0 & 1.000 (Reference) & & & \\
\hline 1 & $1.104(0.664-1.837)$ & 0.703 & & \\
\hline Clinical M stage at diagnosis & & 0.455 & & \\
\hline 0 & 1.000 (Reference) & & & \\
\hline $1 \mathrm{a}$ & $0.638(0.150-2.711)$ & 0.543 & & \\
\hline $1 b$ & $0.662(0.383-1.143)$ & 0.139 & & \\
\hline $1 c$ & $0.997(0.444-2.238)$ & 0.995 & & \\
\hline Definitive local treatment & & 0.519 & & \\
\hline No & 1.000 (Reference) & & & \\
\hline Radical prostatectomy & $1.534(0.762-3.087)$ & 0.231 & & \\
\hline Radiation treatment & $1.205(0.555-2.617)$ & 0.638 & & \\
\hline PSA level at DTX-based chemotherapy induction & $1.000(1.000-1.001)$ & 0.158 & $0.999(0.997-1.001)$ & 0.176 \\
\hline Clinical N stage at DTX-based chemotherapy induction & & 0.643 & & \\
\hline 0 & 1.000 (Reference) & & & \\
\hline 1 & $0.886(0.531-1.479)$ & 0.643 & & \\
\hline Clinical M stage at DTX-based chemotherapy induction & & 0.803 & & \\
\hline $1 a$ & 1.000 (Reference) & & & \\
\hline $1 b$ & $0.709(0.254-1.980)$ & 0.511 & & \\
\hline 1c & $0.706(0.212-2.349)$ & 0.570 & & \\
\hline PSA nadir level responding to DTX & $1.000(1.000-1.001)$ & 0.125 & $1.000(1.000-1.001)$ & 0.187 \\
\hline No. of DTX-based chemotherapy cycles & $0.939(0.902-0.979)$ & 0.003 & $0.934(0.899-0.970)$ & $<0.001^{*}$ \\
\hline
\end{tabular}

PFS: progression-free survival, HR: hazard ratio, Cl: confidence interval, ADT: androgen deprivation therapy, PSA: prostate-specific antigen, DTX: docetaxel.

${ }^{*} p<0.05$.

level at DTX-based chemotherapy induction, clinical $\mathrm{N}$ stage at DTX-based chemotherapy induction, PSA nadir level, and number of DTX-based chemotherapy cycles were included in multivariate analysis. The result obtained through multivariate analysis indicated that the PSA nadir level (HR, 1.001; 95\% CI, 1.000-1.002; $\mathrm{p}<0.001$ ), and number of DTX-based chemotherapy cycles (HR, 0.932; 95\% CI, 0.876-0.991; p=0.024) were significant. 


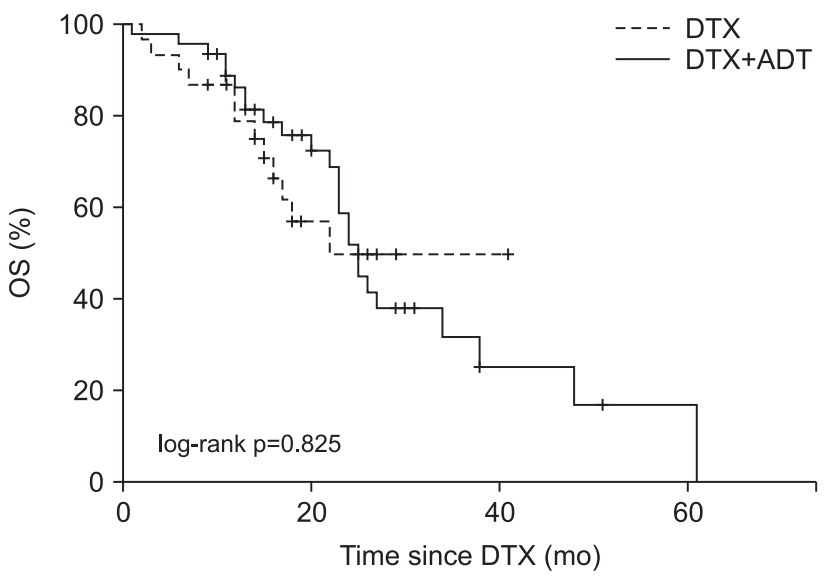

Fig. 2. The dotted line shows the OS of the DTX group, and the linear line shows the OS of the DTX+ADT group. OS: overall survival, DTX: docetaxel, ADT: androgen deprivation therapy.

\section{DISCUSSION}

In our study, concurrent use of DTX and ADT affected the PFS but did not show a significant correlation with the OS. Our results also indicated that the number of DTX dosage was significantly associated with PFS and OS. In addition, there were significant correlation between PSA nadir level and OS.

In newly diagnosed metastatic prostate cancer, there is a rapid response to surgical or medical castration, such as decline in the serum PSA level, regression of metastases at the soft-tissue and improvement in bone pain [11]. ADT as the main treatment in general or adjuvant treatment after radical prostatectomy has not been confirmed as beneficial for localized prostate cancer patients $[12,13]$. There are several studies that have confirmed its beneficial effect in locally advanced and metastatic diseases, and as the main treatment with or without definitive local treatment [14-17]. But ADT can induce various side effects; skeletal complications such as fractures, erectile dysfunction, metabolic diseases such as anemia, diabetes and cardiovascular disease, hot flashes, and mood changes [18]. Moreover, according to the 2008 Medicare data, LHRH agonists had a high cost of approximately $\$ 10$ billion in 2003. The need for ADT should be reconsidered based on its various side effects and costs [19].

Although a majority of patients initially respond to ADT, most will eventually develop castration resistance, defined as disease progression despite serum testosterone levels of $<20 \mathrm{ng} / \mathrm{dL}$. Castration resistance could be due to the persistence of AR signaling under systemic castration via gene mutations, intra-tumoral production of androgens, and multiple alternative survival pathways [20]. Despite continuous hormone manipulation, disease progression to CRPC occurs after a mean time of 2 to 3 years. Subsequently, metastatic CRPC may occur. Poor prognosis is associated with that showing the mean survival time of only 16 to 18 months [21,22].

In patients with CRPC showing disease progression while on the initial ADT regimen, ADT is generally continued in conjunction with secondary therapies [23]. There are no updated randomized trials focused on continued ADT in men with CRPC. A study of 341 CRPC patients concluded that survival benefit of 2 to 6 months was obtained by continued testicular androgen suppression [9]. Therefore, studies of CRPC have focused on continuous ADT.

Until now, only a few studies have investigated the effects of ADT in combination with DTX-based chemotherapy in metastatic CRPC patients. Most of the studies were published in Korea, which may be because of the policy of the Korean national health insurance service, which disallowed the use of ADT during DTXbased chemotherapy. A retrospective study of 21 patients who received DTX-based chemotherapy and 26 patients who received DTX-based chemotherapy with ADT at a single institution was conducted by Jang et al [24]. The primary end points were biochemical PFS (bPFS) and radiographic PFS (rPFS). The median bPFS of 5.0 months in the DTX group and 8.0 months in the DTX+ADT group indicated a significant survival benefit of the combination therapy through Kaplan-Meier analysis (log-rank $\mathrm{p}=0.044$ ). Likewise, the median $\mathrm{rPFS}$ of 6.0 months in the DTX group and 9.0 months in the DTX+ADT group revealed a survival benefit of the combination therapy through Kaplan-Meier analysis (log-rank $\mathrm{p}=0.036$ ). Lee et al [25] conducted a retrospective multicenter study including 44 patients in the DTX group and 28 patients in the DTX+ADT group and assessed survival using Kaplan-Meier analysis. They reported that the combination therapy showed a significant beneficial effect on the bPFS (log-rank $\mathrm{p}=0.024$ ), but not on the rPFS (log-rank $\mathrm{p}=0.387$ ). Another study of Lee et al [26] included 39 patients in the DTX group and 39 patients in the DTX+ADT group. PFS was the primary end point, defined as the time from the start of chemotherapy to the occurrence of either disease progression (PSA progression according 
Table 3. Univariate and multivariate Cox regression analyses of the factors predicting the OS

\begin{tabular}{|c|c|c|c|c|}
\hline \multirow{2}{*}{ Characteristic } & \multicolumn{2}{|c|}{ Univariate analysis } & \multicolumn{2}{|c|}{ Multivariate analysis } \\
\hline & $\mathrm{HR}(95 \% \mathrm{CI})$ & p-value & $\mathrm{HR}(95 \% \mathrm{CI})$ & p-value \\
\hline ADT & $0.924(0.456-1.872)$ & 0.827 & & \\
\hline Age & $0.995(0.956-1.035)$ & 0.802 & & \\
\hline Gleason score & & 0.225 & & 0.408 \\
\hline$\leq 7$ & 1.000 (Reference) & & 1.000 (Reference) & \\
\hline 8 & $0.436(0.108-1.759)$ & 0.243 & $0.450(0.109-1.850)$ & 0.268 \\
\hline 9 & $1.317(0.389-4.467)$ & 0.658 & $1.151(0.333-3.972)$ & 0.824 \\
\hline 10 & $1.224(0.301-4.983)$ & 0.778 & $1.020(0.245-4.236)$ & 0.979 \\
\hline Unknown & $0.909(0.143-5.780)$ & 0.919 & $0.661(0.098-4.458)$ & 0.671 \\
\hline PSA level at diagnosis & $1.000(1.000-1.000)$ & 0.074 & & \\
\hline Clinical T stage at diagnosis & & 0.707 & & \\
\hline 1 & 1.000 (Reference) & & & \\
\hline 2 & $0.768(0.098-5.997)$ & 0.801 & & \\
\hline $3 a$ & $2.095(0.217-20.205)$ & 0.523 & & \\
\hline $3 b$ & $0.920(0.058-14.723)$ & 0.953 & & \\
\hline 4 & $1.263(0.163-9.784)$ & 0.823 & & \\
\hline Unknown & $0.947(0.117-7.663)$ & 0.960 & & \\
\hline Clinical $\mathrm{N}$ stage at diagnosis & & 0.937 & & \\
\hline 0 & 1.000 (Reference) & & & \\
\hline 1 & $1.027(0.529-1.994)$ & 0.937 & & \\
\hline Clinical M stage at diagnosis & & 0.973 & & \\
\hline 0 & 1.000 (Reference) & & & \\
\hline $1 \mathrm{a}$ & $0.707(0.092-5.430)$ & 0.739 & & \\
\hline $1 b$ & $1.017(0.498-2.075)$ & 0.964 & & \\
\hline $1 c$ & $1.188(0.390-3.623)$ & 0.762 & & \\
\hline Definitive local treatment & & 0.915 & & \\
\hline No & 1.000 (Reference) & & & \\
\hline Radical prostatectomy & $1.053(0.422-2.626)$ & 0.911 & & \\
\hline Radiation treatment & $1.414(0.541-3.692)$ & 0.480 & & \\
\hline PSA level at DTX-based chemotherapy induction & $1.001(1.000-1.001)$ & $<0.001$ & & 0.431 \\
\hline Clinical N stage at DTX-based chemotherapy induction & & 0.082 & & 0.062 \\
\hline 0 & 1.000 (Reference) & & & \\
\hline 1 & $1.809(0.927-3.531)$ & 0.082 & & \\
\hline Clinical M stage at DTX-based chemotherapy induction & & 0.399 & & \\
\hline $1 \mathrm{a}$ & 1.000 (Reference) & & & \\
\hline $1 b$ & $0.486(0.144-1.634)$ & 0.244 & & \\
\hline 1c & $0.725(0.168-3.122)$ & 0.666 & & \\
\hline PSA nadir level responding to DTX & $1.001(1.001-1.002)$ & $<0.001$ & $1.001(1.000-1.002)$ & $<0.001^{*}$ \\
\hline No. of DTX-based chemotherapy cycles & $0.928(0.872-0.986)$ & 0.016 & $0.932(0.876-0.991)$ & $0.024^{*}$ \\
\hline
\end{tabular}

OS: overall survival, HR: hazard ratio, Cl: confidence interval, ADT: androgen deprivation therapy, PSA: prostate-specific antigen, DTX: docetaxel. ${ }^{*} \mathrm{p}<0.05$.

to the PCWG criteria, symptomatic deterioration, or radiographic progression) or death, which was different from the definition used in other studies. In their study, the median PFS was 4.9 months in the DTX group and 5.0 months in the DTX+ADT group (HR,
0.85; 95\% CI, 0.53-1.42; $\mathrm{p}=0.57$ ), and the median OS was 22.0 months in the DTX group and 24.8 months in the DTX+ADT group (HR, 0.98; 95\% CI, 0.53-1.78; p=0.94). Additionally, testosterone remained suppressed during the DTX treatment-period, which corroborates the re- 
sults of no significant difference in survival.

Several studies investigated the testosterone levels after withdrawal of ADT. In a retrospective study of 221 patients in Korea, Nam et al [27] reported that serum testosterone levels were recovered to baseline levels in a mean of 6.8 months after cessation of $\mathrm{ADT}$. Age, initial testosterone level and duration of ADT were significantly associated with recovery to supracastration. In a prospective study of 66 patients, Nejat et al [28] reported that median time to baseline testosterone levels after cessation of ADT was 7 months. Similarly, D'Amico et al [29] reported that after 6 and 12 months of ADT cessation, $16 \%$ and $38 \%$ of men showed return to baseline testosterone levels. In these studies, the serum testosterone levels of many patients were recovered to supra-castration or baseline levels in about 7 months. These findings of recovered testosterone levels after withdrawal of ADT could help explain the results of our study.

There have been limited studies about the relation between the number of DTX cycles administered and survival benefit. According to Mainsail study by de Morrée et al [30], total number of DTX cycles administered was an independent factor for OS in metastatic CRPC patients. In this study, the authors reported that more than 10 cycles showed superior OS compared with 8 to 10 cycles or 5 to 7 cycles. More than 10 DTX cycles showed a median OS of 33.0 months compared to 26.9 months of 8 to 10 cycles. These findings are correlated with results of our study showing survival benefit with increasing DTX cycles.

Our study has several limitations. First, most of the data were analyzed retrospectively. Second, limited number of patients were enrolled because of the strict protocols. Third, testosterone data of each patient are lacking. Finally, the differences in the health insurance system between Korea and Japan might be a bias. Nevertheless, our study is an international multicenter study with matched protocols and definitive results.

\section{CONCLUSIONS}

Concurrent DTX-based chemotherapy and ADT may be beneficial compared with DTX-based chemotherapy alone in chemotherapy-naïve metastatic CRPC patients in terms of the PFS, but not the OS. Previous studies as well as the current study had a retrospective design and included a small number of patients. Hence, a ran- domized, prospective study including a large number of patients is needed to validate our findings.

\section{ACKNOWLEDGEMENTS}

This work was supported by the National Research Foundation of Korea (NRF) grant funded by the Korea government (MSIT) (No. NRF-2019R1G1A1011363).

\section{Conflicts of Interest}

The authors have nothing to disclose.

\section{Author Contribution}

Conceptualization: KM. Data curation: KM, MT. Formal analysis: KM, MT. Investigation: JWC, BSK. Methodology: JNL. Project administration: YSH. Resources: HTK, THK. Software: TGK. Supervision: SHC, TK. Validation: SKC, SE. Visualization: ESY. Writing-original draft: KM. Writing-review \& editing: SHC, TK.

\section{Data Sharing Statement}

The data required to reproduce these findings cannot be shared at this time as the data also forms part of an ongoing study.

\section{REFERENCES}

1. Siegel RL, Miller KD, Jemal A. Cancer statistics, 2016. CA Cancer J Clin 2016;66:7-30.

2. Kimura T, Egawa S. Epidemiology of prostate cancer in Asian countries. Int J Urol 2018;25:524-31.

3. Jung KW, Won YJ, Kong HJ, Lee ES; Community of Population-Based Regional Cancer Registries. Cancer statistics in Korea: incidence, mortality, survival, and prevalence in 2015. Cancer Res Treat 2018;50:303-16.

4. Kakehi Y, Sugimoto M, Taoka R; Committee for Establishment of the evidenced-based clinical practice guideline for prostate cancer of the Japanese Urological Association. Evidenced-based clinical practice guideline for prostate cancer (summary: Japanese Urological Association, 2016 edition). Int J Urol 2017;24:648-66.

5. Scher HI, Halabi S, Tannock I, Morris M, Sternberg CN, Carducci MA, et al.; Prostate Cancer Clinical Trials Working Group. Design and end points of clinical trials for patients with progressive prostate cancer and castrate levels of testos- 
terone: recommendations of the Prostate Cancer Clinical Trials Working Group. J Clin Oncol 2008;26:1148-59.

6. Petrylak DP, Tangen CM, Hussain MH, Lara PN Jr, Jones JA, Taplin ME, et al. Docetaxel and estramustine compared with mitoxantrone and prednisone for advanced refractory prostate cancer. N Engl J Med 2004;351:1513-20.

7. Tannock IF, de Wit R, Berry WR, Horti J, Pluzanska A, Chi $\mathrm{KN}$, et al. Docetaxel plus prednisone or mitoxantrone plus prednisone for advanced prostate cancer. N Engl J Med 2004; 351:1502-12.

8. Hussain M, Wolf M, Marshall E, Crawford ED, Eisenberger M. Effects of continued androgen-deprivation therapy and other prognostic factors on response and survival in phase II chemotherapy trials for hormone-refractory prostate cancer: a Southwest Oncology Group report. J Clin Oncol 1994;12: 1868-75.

9. Taylor CD, Elson P, Trump DL. Importance of continued testicular suppression in hormone-refractory prostate cancer. J Clin Oncol 1993;11:2167-72.

10. Scher HI, Buchanan G, Gerald W, Butler LM, Tilley WD. Targeting the androgen receptor: improving outcomes for castration-resistant prostate cancer. Endocr Relat Cancer 2004;11:459-76.

11. Crawford ED, Eisenberger MA, McLeod DG, Spaulding JT, Benson R, Dorr FA, et al. A controlled trial of leuprolide with and without flutamide in prostatic carcinoma. N Engl J Med 1989;321:419-24.

12. Wong YN, Freedland S, Egleston B, Hudes G, Schwartz JS, Armstrong K. Role of androgen deprivation therapy for nodepositive prostate cancer. J Clin Oncol 2009;27:100-5.

13. Lu-Yao GL, Albertsen PC, Moore DF, Shih W, Lin Y, DiPaola RS, et al. Survival following primary androgen deprivation therapy among men with localized prostate cancer. JAMA 2008;300:173-81.

14. Sharifi N, Gulley JL, Dahut WL. Androgen deprivation therapy for prostate cancer. JAMA 2005;294:238-44.

15. Messing EM, Manola J, Sarosdy M, Wilding G, Crawford ED, Trump D. Immediate hormonal therapy compared with observation after radical prostatectomy and pelvic lymphadenectomy in men with node-positive prostate cancer. N Engl J Med 1999;341:1781-8.

16. Messing EM, Manola J, Yao J, Kiernan M, Crawford D, Wilding G, et al.; Eastern Cooperative Oncology Group study EST 3886. Immediate versus deferred androgen deprivation treatment in patients with node-positive prostate cancer after radical prostatectomy and pelvic lymphadenectomy. Lancet Oncol 2006;7:472-9.

17. Bolla M, Collette L, Blank L, Warde P, Dubois JB, Mirimanoff
$\mathrm{RO}$, et al. Long-term results with immediate androgen suppression and external irradiation in patients with locally advanced prostate cancer (an EORTC study): a phase III randomised trial. Lancet 2002;360:103-6.

18. Holzbeierlein JM, McLaughlin MD, Thrasher JB. Complications of androgen deprivation therapy for prostate cancer. Curr Opin Urol 2004;14:177-83.

19. Weight CJ, Klein EA, Jones JS. Androgen deprivation falls as orchiectomy rates rise after changes in reimbursement in the U.S. Medicare population. Cancer 2008;112:2195-201.

20. Karantanos T, Corn PG, Thompson TC. Prostate cancer progression after androgen deprivation therapy: mechanisms of castrate resistance and novel therapeutic approaches. Oncogene 2013;32:5501-11

21. Harris WP, Mostaghel EA, Nelson PS, Montgomery B. Androgen deprivation therapy: progress in understanding mechanisms of resistance and optimizing androgen depletion. Nat Clin Pract Urol 2009;6:76-85.

22. Feldman BJ, Feldman D. The development of androgenindependent prostate cancer. Nat Rev Cancer 2001;1:34-45.

23. Virgo KS, Basch E, Loblaw DA, Oliver TK, Rumble RB, Carducci MA, et al. Second-line hormonal therapy for men with chemotherapy-naïve, castration-resistant prostate cancer: American Society of Clinical Oncology provisional clinical opinion. J Clin Oncol 2017;35:1952-64.

24. Jang HS, Koo KC, Cho KS, Chung BH. Survival outcomes of concurrent treatment with docetaxel and androgen deprivation therapy in metastatic castration-resistant prostate cancer. Yonsei Med J 2016;57:1070-8.

25. Lee DH, Kim JH, Seo WI, Nam JK, Kim TN, Oh CK, et al. Clinical outcomes of continuous addition of androgen deprivation therapy during docetaxel chemotherapy for patients with castration-resistant prostate cancer. Korean J Urol Oncol 2017;15:59-65.

26. Lee JL, Eun Kim J, Ahn JH, Lee DH, Lee J, Kim CS, et al. Role of androgen deprivation treatment in patients with castration-resistant prostate cancer, receiving docetaxel-based chemotherapy. Am J Clin Oncol 2011;34:140-4.

27. Nam W, Choi SY, Yoo SJ, Ryu J, Lee J, Kyung YS, et al. Factors associated with testosterone recovery after androgen deprivation therapy in patients with prostate cancer. Investig Clin Urol 2018;59:18-24.

28. Nejat RJ, Rashid HH, Bagiella E, Katz AE, Benson MC. A prospective analysis of time to normalization of serum testosterone after withdrawal of androgen deprivation therapy. J Urol 2000;164:1891-4.

29. D’Amico AV, Renshaw AA, Loffredo B, Chen MH. Duration of testosterone suppression and the risk of death from pros- 
tate cancer in men treated using radiation and 6 months of hormone therapy. Cancer 2007;110:1723-8.

30. de Morrée ES, Vogelzang NJ, Petrylak DP, Budnik N, Wiechno PJ, Sternberg CN, et al. Association of survival benefit with docetaxel in prostate cancer and total number of cycles administered: a post hoc analysis of the mainsail study. JAMA Oncol 2017;3:68-75. 